\title{
BPSK Modulation with Unequal Power Allocation Considering A Priori Knowledge
}

\author{
Thomas Brüggen, Marc Adrat ${ }^{1}$, and Peter Vary \\ Institute of Communication Systems and Data Processing (ind), RWTH Aachen University, Germany
}

\{brueggen,marc,vary\}@ind.rwth-aachen.de

\begin{abstract}
A well known technique to protect bits with different sensitivities against channel errors is unequal error protection (UEP) by selective channel coding. However, some transmission systems such as Bluetooth and DECT include only weak or no channel coding. For those systems modulation with unequal power allocation (MUPA) is an appropriate technique to realize UEP without channel coding. MUPA allocates different transmission power to the modulation symbols according to the individual bit error sensitivities. In this paper we apply the MUPA concept to systems with softbit source decoding and soft demodulation, and enhance the MUPA approach by taking a $1^{\text {st }}$ order Markov model in terms of $1^{\text {st }}$ order a priori knowledge (AK1) into account (MUPA-AK1).
\end{abstract}

\section{INTRODUCTION}

Digital communication systems transmit the digital representations of the source encoded parameters of speech, audio, or video signals by bits which exhibit individual bit error sensitivities. The most significant bits (MSBs) are more sensitive than the least significant bits (LSBs). At the receiver an MSB, which has been inverted by channel noise, causes a larger distortion in the output signal than an inverted LSB. For this reason, unequal error protection (UEP) is applied and often realized by selective channel coding [1] protecting the MSBs stronger against bit errors than the LSBs.

Due to the higher effort for encoding and decoding, UEP by channel coding is not specified for systems such as Bluetooth and DECT, which are designed to ensure low costs. The modulation with unequal power allocation (MUPA) [2][3][4] achieves UEP by periodically allocating unequal transmission power to the individual bits. Less energy is assigned to the LSB than to the MSB, while the average transmitted energy per bit remains the same. For this purpose, a set of weights $w_{i}$ is calculated once in advance for a representative selection of channel signal-to-noise ratios (SNRs). Before BPSK modulation, the individual bits are weighted differently at the transmitter taking the channel SNR into account. This multiplicative weighting causes only a small additional effort.

MUPA for BPSK modulation has already been described in [3] and [4]. In these proposals MUPA is only used for BPSK systems with hard decision decoding at the receiver. In [2] the MUPA concept has been transferred to a transmission system using a 16-QAM scheme and exploiting a priori knowledge $(\mathrm{AK})$ at the receiver. However, the MUPA technique for the 16-QAM system in [2] does not utilize the autocorrelation of the source encoded parameters. In contrast to [2], [3] and

\footnotetext{
${ }^{1}$ Marc Adrat is now with FGAN e.V., FKIE/KOM, Wachtberg, Germany.
}

[4], in this paper we apply the MUPA concept for the first time to BPSK systems which exploit different orders of AK at the receiver with softbit source decoding (SBSD) [5] or soft demodulation (SDM). In addition, we present a novel approach which enhances MUPA at the transmitter by considering a $1^{\text {st }}$ order Markov model of the source encoded parameters. The resulting approach is called MUPA-AK1 (a priori knowledge of $1^{\text {st }}$ order). In contrast to the MUPA concept described in [4], [3] and [2], MUPA-AK1 takes the autocorrelation of the source encoded parameters into account for the calculation of the weights $w_{i}$.

In [6] the technique of differently weighted bits has already been discussed for PCM transmission. However, in [6] an approximate solution is derived which is based on the simplifying assumption that only single bit errors occur. In [7] UEP is achieved by bit-wise control of the BPSK modulation amplitudes. In contrast to our proposal, neighborhood relations between the unquantized and quantized source parameters are exploited in combination with Gray or Gray-like bit mappings to calculate the discrete symbol amplitudes. In contrast to [6] and [7], MUPA and MUPA-AK1 are analytical approaches with rigorous optimization of the weights $w_{i}$ allowing continuous BPSK symbol amplitudes, arbitrary bit mappings, and taking multiple bit errors into account. Besides, the MUPAAK1 technique developed in this paper considers $\mathrm{AK} 1$ at the receiver and enhances the basic MUPA concept.

\section{SYSTEM MODEL}

The model of the transmission system is shown in Fig. 1. Instead of any specific signal and source encoder, we use a zero-mean Gaussian source with variance $\sigma_{u}^{2}=1$ to model the parameters $u(\tau)$ which are delivered at time $\tau$ by the source encoder. This allows us to conduct precisely defined experiments. However, without loss of generality the results can be applied to any real source encoded for speech, audio, or video signals. Each $u(\tau)$ is quantized to $\bar{u}(\tau) \in\left\{\bar{u}_{\kappa} \mid \kappa=1,2, \ldots 2^{M}\right\}$ with the reproduction levels $\bar{u}_{\kappa}$. The index assignment (IA) maps the values $\bar{u}(\tau)$ by the one-to-one mapping function $\Gamma$ to bit patterns $\underline{x}(\tau)=\Gamma(\bar{u}(\tau))$ of the bit pattern set $\mathcal{Q}=\left\{\underline{x}_{\kappa} \mid \kappa=1,2, \ldots 2^{M}\right\}$ with $\underline{x}_{\kappa}=\left(x_{\kappa}^{(1)}, x_{\kappa}^{(2)}, \ldots x_{\kappa}^{(M)}\right)$. The bits $x_{\kappa}^{(i)} \in\{-1,+1\}, i=1,2, \ldots M$, contain the MSB $x_{\kappa}^{(1)}$ and the LSB $x_{\kappa}^{(M)}$. Each value $x_{\kappa}^{(i)}$ is multiplied with the specific weight $w_{i} \in \mathbb{R}^{+}$(Sec. IV) of the diagonal matrix $\underline{W}=\operatorname{diag}\left(w_{1}, w_{2}, \ldots w_{M}\right)$. A vector of BPSK channel symbols

$$
\underline{y}(\tau)=\underline{W} \cdot \underline{x}(\tau) \text {. }
$$




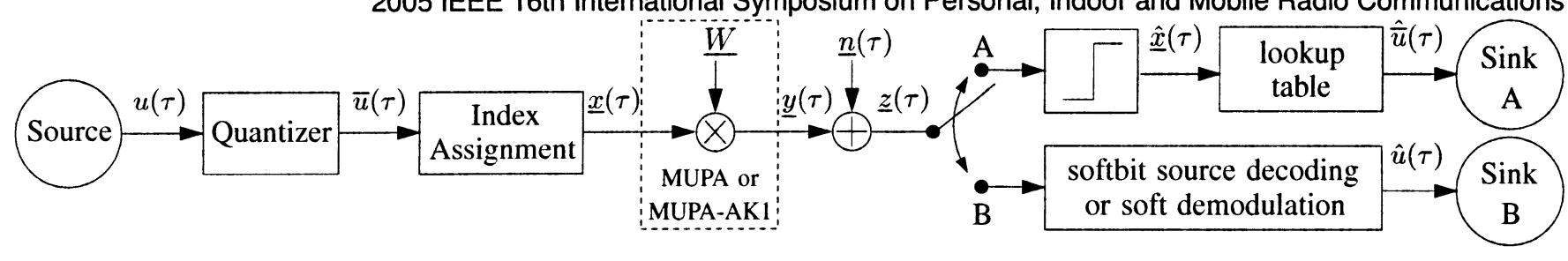

Fig. 1. Baseband system model of BPSK-MUPA with weight matrix $\underline{W}, \mathrm{~A}$ : conventional hard decision decoding, B: soft decoding

with $\underline{y}(\tau) \in\left\{\underline{y}_{\kappa} \mid \kappa=1,2, \ldots 2^{M}\right\}$ and the channel symbols $\underline{y}_{\kappa}=\left(y_{\kappa}^{(1)}, y_{\kappa}^{(2)}, \ldots y_{\kappa}^{(M)}\right), y_{\kappa}^{(i)} \in\left\{-w_{i},+w_{i}\right\}, i=1,2, \ldots M$, is obtained. Each $y_{\kappa}^{(i)}$ represents the bit at position $i$ of a specific $\underline{x}(\tau)=\underline{x}_{\kappa}$ with BPSK. The symbols are transmitted over a channel with additive white Gaussian noise $(\mathrm{AWGN}) \underline{n}(\tau)=\left(n^{(1)}(\tau), n^{(2)}(\tau), \ldots n^{(M)}(\tau)\right), n^{(i)}(\tau) \in \mathbb{R}$, $i=1,2, \ldots M$, with $N\left(0, \sigma_{n}^{2}\right)$, the variance $\sigma_{n}^{2}=N_{0} / 2$, and the noise power spectral density $N_{0}$. We assume perfect knowledge of $\sigma_{n}^{2}$ at the transmitter.

In case of coherent BPSK modulation we obtain $\underline{z}(\tau)=\underline{y}(\tau)+\underline{n}(\tau)$ as disturbed vector at the receiver with $\left.\underline{z}(\tau)=\overline{(}^{(1)}(\tau), z^{(2)}(\tau), \ldots z^{(M)}(\tau)\right)$. If the switch of Fig. 1 is in position $\mathrm{A}$, hard decision decoding is performed and the estimate $\hat{\bar{u}}(\tau) \in\left\{\bar{u}_{\kappa} \mid \kappa=1,2, \ldots 2^{M}\right\}$ is produced by table lookup. If the switch is in position B, SBSD or SDM is utilized to estimate the output value $\hat{u}(\tau) \in \mathbb{R}$. In this paper we focus on SBSD and SDM. Hard decision decoding is only used to calculate the weights for MUPA-AK1 and as reference to clarify performance improvements.

\section{SOFT Demodulation (SDM)}

In the following we shortly review the concept of soft demodulation for BPSK, which was developed in [2] for 16QAM and is based on softbit source decoding (SBSD). For a detailed derivation of SBSD we refer to the literature [5].

The soft estimation of $\hat{u}(\tau)$ at the receiver requires a posteriori probabilities providing information about all possibly transmitted bit patterns $\underline{x}(\tau) \in \mathcal{Q}$ given the received $\underline{z}(\tau)$. With a $1^{\text {st }}$ order Markov model for the source encoded parameters and a memoryless channel, the a posteriori probabilities $P\left(\underline{y}_{\kappa}(\tau) \mid \underline{z}(\tau), \underline{Z}(\tau-1)\right)$ can be calculated as

$$
\begin{aligned}
P\left(\underline{y}_{\kappa}(\tau)\right. & \mid \underline{z}(\tau), \underline{Z}(\tau-1)) \\
& =C \cdot p\left(\underline{z}(\tau) \mid \underline{y}_{\kappa}(\tau)\right) \cdot P\left(\underline{y}_{\kappa}(\tau) \mid \underline{Z}(\tau-1)\right)
\end{aligned}
$$

with $\underline{Z}(\tau-1)=(\underline{z}(\tau-1), \underline{z}(\tau-2) \ldots)$ consisting of the history of received values from the beginning of the transmission until the time instant $\tau-1$. $C$ is a normalization constant.

For SBSD the bit-wise estimation of the parameter transition probability $p\left(\underline{z}(\tau) \mid \underline{y}_{\kappa}(\tau)\right)$ can be accomplished by the so-called $L$ values. In contrast to SBSD, in SDM the Gaussian probability for the absolute distance between the received symbol $z^{(i)}(\tau)$ and the transmitted BPSK modulated symbol $y_{\kappa}^{(i)}(\tau) \in\left\{-w_{i},+w_{i}\right\}$ is taken for $p\left(\underline{z}(\tau) \mid \underline{y}_{\kappa}(\tau)\right)$. We consider the real-valued Euclidean distance $D_{z^{(i)}, y^{(i)}}^{2}=\left|z^{(i)}(\tau)-y_{\kappa}^{(i)}(\tau)\right|^{2}$ between the received value $z^{(i)}$ and the possibly sent value $y_{\kappa}^{(i)}(\tau)$ for the bit at position $i$. The AWGN of the channel causes the distance $D_{z^{(i)}, y^{(i)}}^{2}$ and the conditional probability density $p\left(z^{(i)} \mid y_{\kappa}^{(i)}\right)$ becomes

$$
\begin{aligned}
p\left(z^{(i)}(\tau) \mid y_{\kappa}^{(i)}(\tau)\right) & =p\left(D_{z^{(i)}, y^{(i)}}\right) \\
& =\frac{1}{\sqrt{2 \pi} \sigma_{n}} \cdot \exp \left(\frac{-D_{z^{(i)}, y^{(i)}}^{2}}{2 \sigma_{n}^{2}}\right) .
\end{aligned}
$$

With (3) the parameter transition probability is

$$
p\left(\underline{z}(\tau) \mid \underline{y}_{\kappa}(\tau)\right)=\prod_{i=1}^{M} p\left(z^{(i)}(\tau) \mid y_{\kappa}^{(i)}(\tau)\right)
$$

with $\kappa=1,2, \ldots 2^{M}$.

The probabilities $P\left(\underline{y}_{\kappa}(\tau) \mid Z(\tau-1)\right)$ in (2) are determined with the available a priori knowledge. If no a priori knowledge (NAK) is used, the probability density $p\left(\underline{z}(\tau) \mid \underline{y}_{\kappa}(\tau)\right)$ of (4) is proportional to the a posteriori probability [5]:

$$
P\left(\underline{y}_{\kappa}(\tau) \mid \underline{z}(\tau)\right)=C \cdot p\left(\underline{z}(\tau) \mid \underline{y}_{\kappa}(\tau)\right)
$$

with the normalization constant

$$
C=\frac{1}{\sum_{k=1}^{2^{M}} p\left(\underline{z}(\tau) \mid \underline{y}_{k}(\tau)\right)} .
$$

In the following we will use the factor $C$ to normalize the a posteriori probabilities $P\left(\underline{y}_{\kappa}(\tau) \mid \underline{z}(\tau), \ldots\right)$ such that $\sum_{\kappa} P\left(\underline{y}_{\kappa}(\tau) \mid \underline{z}(\tau), \ldots\right)=1$ is fulfilled. Note, that $C$ does not have to equal the term given in (6) in all cases.

With a priori knowledge of $0^{\text {th }}$ order (AK0, i.e., histogram knowledge), the probabilities of occurrence $P\left(y_{\kappa, \tau}\right)$ of the signal points $y_{\kappa, \tau} \in \mathcal{Y}$ determine the a posteriori probabilities. Since $\Gamma$ is a one-to-one mapping function, it holds

$$
P\left(\underline{y}_{\kappa}(\tau)\right)=P\left(\Gamma\left(\bar{u}_{\kappa}(\tau)\right)\right)
$$

with the probabilities of occurrence $P\left(\bar{u}_{\kappa}(\tau)\right), \kappa=1,2, \ldots 2^{M}$, of the quantizer reproduction levels $\bar{u}_{\kappa}(\tau)$ mapped to the symbols $y_{\kappa}(\tau) \in \mathcal{Y}$. For (2) it holds

$$
P\left(\underline{y}_{\kappa}(\tau) \mid z(\tau)\right)=C \cdot p\left(z(\tau) \mid \underline{y}_{\kappa}(\tau)\right) \cdot P\left(\Gamma\left(\bar{u}_{\kappa}(\tau)\right)\right)
$$

For $1^{\text {st }}$ order a priori knowledge (AK1) the correlation of the source parameters $u_{\tau}$ (Fig. 1) is exploited. The a posteriori probabilities can be calculated recursively, because the source parameters can be modelled as a $1^{\text {st }}$ order Markov process [5]:

$$
\begin{aligned}
& P\left(\underline{y}_{\kappa}(\tau) \mid z(\tau), Z(\tau-1)\right)=C \cdot p\left(z(\tau) \mid \underline{y}_{\kappa}(\tau)\right) \\
& \cdot \sum_{\vartheta=1}^{2^{M}} P\left(\underline{y}_{\kappa}(\tau) \mid \underline{y}_{\vartheta}(\tau-1)\right) \cdot P\left(\underline{y}_{\vartheta}(\tau-1) \mid z(\tau-1), Z(\tau-2)\right) .
\end{aligned}
$$


The minimum mean square error (MMSE) between $\bar{u}_{\kappa}$ and $\hat{u}_{\tau}$ is an appropriate and established error criterion $[5,8]$. The last step of the soft demodulation algorithm is the mean square (MS) estimator [5]:

$$
\hat{u}(\tau)=\sum_{\kappa=1}^{2^{M}} \bar{u}_{\kappa} \cdot P\left(\Gamma^{-1}\left(\underline{y}_{\kappa}(\tau)\right) \mid \underline{z}(\tau), \ldots\right) .
$$

\section{BPSK With UNEQUal POWER Allocation}

In the following we assume ergodicity and omit the time index $\tau$ for convenience. We consider the bipolar channel symbols $\pm \sqrt{E_{b}^{(i)}}$ with $E_{b}^{(i)}$ being the energy for each bit $i$. If bits in $\underline{x}_{\kappa}$ are inverted due to the AWGN, the specific distortion $d_{\kappa, \eta}=\bar{u}_{\kappa}-\hat{u}_{\eta}$ occurs. The basic MUPA approach [4],[3],[2] is based on the mean squared error (MSE)

$$
\mathrm{E}\left\{d^{2}\right\}=\sum_{\kappa=1}^{2^{M}} \sum_{\eta=1}^{2^{M}} d_{\kappa, \eta}^{2} \cdot P\left(\underline{x}_{\kappa}\right) \cdot P\left(\underline{\hat{x}}_{\eta} \mid \underline{x}_{\kappa}\right)
$$

with the probability of occurrence $P\left(\underline{x}_{\kappa}\right)$ and the transition probabilities $P\left(\underline{\hat{x}}_{\eta} \mid \underline{x}_{\kappa}\right)$ between the transmitted and received bit patterns $\underline{x}_{\kappa}$ and $\underline{\hat{x}}_{\eta}$, respectively. MUPA with all $w_{i}=1.0$, $i=1, \ldots M$, is identical to BPSK with equal bit error rates (BER) $P_{b}$ for all bits [9]

$$
P_{b}=\frac{1}{2} \operatorname{erfc}\left(\sqrt{\frac{E_{b}}{N_{0}}}\right)=\int_{\sqrt{E_{b}}}^{\infty} \frac{1}{\sqrt{2 \pi} \sigma_{n}} \cdot \exp \left(\frac{-\xi^{2}}{2 \sigma_{n}^{2}}\right) \mathrm{d} \xi .
$$

$P_{b}$ depends on the bit energy $E_{b}$. With MUPA, the $M$ different bits are transmitted with different bit energies $E_{b}^{(i)}=w_{i}^{2} \cdot E_{b}$, $i=1, \ldots M$, according to their bit error sensitivities. If $w_{i}>1$, the bit error probability is reduced due to the higher amplitude, i.e., higher energy for bit $i$. Otherwise, if $w_{i}<1$, the BER is increased. With the normalization of the average energy per bit $E_{b}=1$ the different bit error probabilities are

$$
P_{b}^{(i)}=\int_{w_{i}}^{\infty} \frac{1}{\sqrt{2 \pi} \sigma_{n}} \cdot \exp \left(\frac{-\xi^{2}}{2 \sigma_{n}^{2}}\right) \mathrm{d} \xi .
$$

The approach (11) includes the probability of occurrence $P\left(\underline{x}_{\kappa}\right)$ (i.e., AK0), but no autocorrelation of the source encoded parameters (i.e., AK1). However, in case of residual parameter correlation, this $1^{\text {st }}$ order a priori knowledge is significant information, which can be exploited at the receiver by SBSD or SDM. Consequently, it might be beneficial to extend the approach (11) by $1^{\text {st }}$ a priori knowledge (AK1) to MUPA-AK1. This technique is a signal processing step at the transmitter site and allows to support SBSD and SDM with $\mathrm{AK} 1$ at the receiver site to increase system robustness.
SBSD and SDM calculate the estimated value $\hat{u}$ from $\underline{z}$ by soft decoding. However, for the computation of the weights, we restrict our following considerations to hard decision $\hat{u}=\hat{\bar{u}}()$ (switch in position A, Fig. 1) to keep the effort manageable. These weights will then be used in the system with SDM or SBSD (switch in position B, Fig. 1). The difference between (11) and the novel extended approach developed in the following is the consideration of AK1, i.e., $P(\underline{x}(\tau) \mid \underline{x}(\tau-1))$. From now on, we denote the bit pattern at the previous time instant $\tau-1$ by the additional index "-": $\underline{x}_{\kappa-} \in \mathcal{Q}$ for the bit pattern transmitted at $\tau-1$ and $\underline{\hat{x}}_{\eta-} \in \mathcal{Q}$ for the estimated one.

We assume, that the MSE E $\left\{d^{2}\right\}$ of MUPA-AK1 depends on the transmitted and the estimated bit pattern at time $\tau$, i.e., $\underline{x}_{\kappa}$ and $\underline{\hat{x}}_{\eta}$, respectively, and on the transmitted and the estimated bit pattern at $\tau-1$, i.e., $\underline{x}_{\kappa-}$ and $\underline{\hat{x}}_{\eta-}$, respectively. However, it can be shown that $\mathrm{E}\left\{d^{2}\right\}$ is independent from $\underline{\hat{x}}_{\eta-}$ due to $\sum_{\eta-=1}^{2^{M}} P\left(\underline{\hat{x}}_{\eta-} \mid \underline{x}_{\kappa-}\right)=1$. Consequently, instead of (11) we have:

$$
\mathrm{E}\left\{d^{2}\right\}=\sum_{\kappa=1}^{2^{M}} \sum_{\eta=1}^{2^{M}} \sum_{\kappa-=1}^{2^{M}} d_{\kappa, \eta, \kappa-}^{2} \cdot P\left(\underline{\hat{x}}_{\eta}, \underline{x}_{\kappa}, \underline{x}_{\kappa-}\right)
$$

with the squared distortion

$$
d_{\kappa, \eta, \kappa-}^{2}=\left(\bar{u}_{\kappa}-\hat{u}_{\eta, \kappa-}\right)^{2} .
$$

The estimated value $\hat{u}_{\eta, \kappa-}$, which depends on the presently estimated $\underline{\hat{x}}_{\eta}$ and on the previously sent $\underline{x}_{\kappa-}$, is the result of an MS estimator as used in SBSD and SDM, which calculates the expected value over all possibly transmitted quantizer reproduction levels $\bar{u}_{m}, m=1,2, \ldots M$ :

$$
\hat{u}_{\eta, \kappa-}=\sum_{m=1}^{2^{M}} \bar{u}_{m} \cdot P\left(\underline{x}_{m} \mid \underline{\hat{x}}_{\eta}, \underline{x}_{\kappa-}\right)
$$

with $\underline{x}_{m}=\Gamma\left(\bar{u}_{m}\right) \in \mathcal{Q}$. We apply the Bayes theorem and the chain rule for probabilities to $P\left(\underline{x}_{m} \mid \underline{\hat{x}}_{\eta}, \underline{x}_{\kappa-}\right)$. With $P\left(\underline{\hat{x}}_{\eta} \mid \underline{x}_{m}, \underline{x}_{\kappa-}\right)=P\left(\underline{\hat{x}}_{\eta} \mid \underline{x}_{m}\right)$ due to the memoryless channel and $P\left(\underline{x}_{m} \mid \underline{x}_{\kappa-}\right)=P\left(\underline{x}_{m} \mid \underline{x}_{\kappa-}\right)$ due to the $1^{\text {st }}$ order Markov property, it holds

$$
P\left(\underline{x}_{m} \mid \underline{\hat{x}}_{\eta}, \underline{x}_{\kappa-}\right)=\frac{P\left(\underline{\hat{x}}_{\eta} \mid \underline{x}_{m}\right) \cdot P\left(\underline{x}_{m} \mid \underline{x}_{\kappa-}\right)}{\sum_{n=1}^{2^{M}} P\left(\underline{\hat{x}}_{\eta} \mid \underline{x}_{n}\right) \cdot P\left(\underline{x}_{n} \mid \underline{x}_{\kappa-}\right)} .
$$

Analogously, the joint probability in (14) can be splitted with the chain rule into the probabilities:

$$
P\left(\underline{\hat{x}}_{\eta}, \underline{x}_{\kappa}, \underline{x}_{\kappa-}\right)=P\left(\underline{\hat{x}}_{\eta} \mid \underline{x}_{\kappa}\right) \cdot P\left(\underline{x}_{\kappa} \mid \underline{x}_{\kappa-}\right) \cdot P\left(\underline{x}_{\kappa-}\right) .
$$

We combine (17), (18), (19) and (14) to the MSE with $1^{\text {st }}$ order a priori knowledge, the MUPA-AK1 approach (16).

$$
\mathrm{E}\left\{d^{2}\right\}=\sum_{\kappa=1}^{2^{M}} \sum_{\eta=1}^{2^{M}} \sum_{\kappa=-1}^{2^{M}}\left(\bar{u}_{\kappa}-\frac{\sum_{m=1}^{2^{M}} \bar{u}_{m} \cdot P\left(\underline{\hat{x}}_{\eta} \mid \underline{x}_{m}\right) \cdot P\left(\underline{x}_{m} \mid \underline{x}_{\kappa-}\right)}{\sum_{n=1}^{2^{M}} P\left(\underline{x}_{\eta} \mid \underline{x}_{n}\right) \cdot P\left(\underline{x}_{n} \mid \underline{x}_{\kappa-}\right)}\right)^{2} \cdot P\left(\underline{\hat{x}}_{\eta} \mid \underline{x}_{\kappa}\right) \cdot P\left(\underline{x}_{\kappa} \mid \underline{x}_{\kappa-}\right) \cdot P\left(\underline{x}_{\kappa-}\right)
$$


As the noise samples are statistically independent, the channel transition probabilities can be expressed as [4],[9],[10]

$$
P\left(\underline{\hat{x}}_{\eta} \mid \underline{x}_{s}\right)=\left(\prod_{\substack{i=1 \\ x_{s}^{(i)} \neq \hat{x}_{\eta}^{(i)}}}^{M} P_{b}^{(i)}\right) \cdot\left(\prod_{\substack{i=1 \\ x_{s}^{(i)}=\hat{x}_{\eta}^{(i)}}}^{M}\left(1-P_{b}^{(i)}\right)\right)
$$

with $s \in\{m, n, \kappa\}$ and $P_{b}^{(i)}$ from (13).

In most transmission systems the energy budget is limited or additional signal energy increases interference as, e.g., in code division multiple access (CDMA) systems. We restrict the transmission energy by normalizing the average bit energy $E_{b}$ to 1 . With the different bit energies $E_{b}^{(i)}=w_{i}^{2}$ the energy constraint is

$$
\sum_{i=1}^{M} w_{i}^{2}=M
$$

\section{A. Lagrange Multiplier Method (LMM)}

Our goal is to minimize the MSE E $\left\{d^{2}\right\}$ of (16) by optimizing the weights $w_{i}$ under the constraint (21). An appropriate approach to find a solution for this optimization problem is the Langrange Multiplier Method (LMM) with the Lagrange Multiplier $\lambda$. The Lagrange equation is built by the MSE (16) and the energy condition (21):

$$
L=\mathrm{E}\left\{d^{2}\right\}-\lambda\left(M-\sum_{i=1}^{M} w_{i}^{2}\right) .
$$

This equation is a non-linear function $L: \mathbb{R}^{M+1} \rightarrow \mathbb{R}$ with $M+1$ unknown variables, i.e., the $M$ weights $w_{i}$ and the Lagrange Multiplier $\lambda$. Next, we need the $M+1$ partial derivatives $\partial L\left(w_{1}, \ldots w_{M}, \lambda\right) / \partial w_{j}, j=1,2, \ldots M$, and $\partial L\left(w_{1}, \ldots w_{M}, \lambda\right) / \partial \lambda$ of (22). Note, that only the channel transition probabilities $P\left(\underline{\hat{x}}_{\eta} \mid \underline{x}_{s}\right), s \in\{m, n, \kappa\}$, depend on $w_{i}$ and have to be differentiated with respect to $w_{j}$.

\section{B. Newton Algorithm}

In the next LMM step the roots $w_{j}$ and $\lambda$ of the $M+1$ derivatives have to be calculated. Due to the transcendent function $\exp (x)$, we employ the $(M+1)$-dimensional Newton algorithm to calculate the roots. For convenience, we insert $\lambda$ into the set of variables by renaming $\left(w_{1}, \ldots w_{M}, \lambda\right)^{T}$ to $\underline{v}=\left(v_{1}, \ldots v_{M+1}\right)^{T}$ with $v_{i}=w_{i}$ for $i=1,2, \ldots M$, and $v_{M+1}=\lambda$. We define $L_{j}^{\prime}(\underline{v})=\partial L(\underline{v}) / \partial v_{j}$, $j=1,2, \ldots M+1$, and $\underline{L}^{\prime}(\underline{v})=\left(L_{1}^{\prime}(\underline{v}), \ldots L_{M+1}^{\prime}(\underline{v})\right)^{T}$ for the vector of the first partial derivatives. With this notation, the Newton iteration rule is given by

$$
\underline{v}_{k+1}=\underline{v}_{k}-J_{L}^{-1}\left(\underline{v}_{k}\right) \cdot \underline{L}^{\prime}\left(\underline{v}_{k}\right)
$$

with the iteration counter $k$ and the inverse Jacobian matrix $J_{L}^{-1}\left(\underline{v}_{k}\right)$ containing the first partial derivatives of $L^{\prime}(\underline{v})$, i.e., the second partial derivatives of $L(\underline{v}), L^{\prime \prime}(\underline{v})=\partial L_{g}^{\prime}(\underline{v}) / \partial v_{j}$, for all combinations of $g, j=1,2, \ldots M+1$.

When the exit condition $\left\|\underline{v}_{k+1}-\underline{v}_{k}\right\|<\epsilon$ (here: $\epsilon=10^{-10}$ ) is fulfilled, the LMM supplies the weights $w_{i}$ as optimized solution. It is very difficult to check this solution for minimum analytically due to the non-linearity of (16) and (21). For simplification, we take other sets of weights with $\breve{w}_{i}$ fulfilling (21) and which are close to $w_{i}$. If the $\mathrm{E}\left\{d^{2}\right\}$ of (16) is larger with the $\breve{w}_{i}$ than with the $w_{i}$, we suppose that the optimized $w_{i}$ are the solution for a local or a global minimum. The simulation results (Sec. V) show that this strategy is successful.

\section{Simulation Results}

The new MUPA-AK1 approach is compared with the basic MUPA approach [4],[3] and with systems with constant symbol energy. All simulations are carried out with

- a source signal with zero-mean Gaussian probability density function (pdf), the variance $\sigma_{u}^{2}=1$, and correlated source parameters $(a=0.9)$,

- a symmetric Lloyd-Max quantizer (LMQ) which is pdfoptimized for the source signal $u$,

- index assignment $\Gamma$ : Gray

- BPSK modulation with SDM or SBSD, and AK1 or AK0, respectively.

The simulation results are evaluated in terms of the parameter signal-to-noise ratio (SNR)

$$
\mathrm{SNR}[\mathrm{dB}]=10 \log _{10}\left(\frac{\mathrm{E}\left\{\mathrm{u}^{2}(\tau)\right\}}{\mathrm{E}\left\{(\mathrm{u}(\tau)-\hat{\mathrm{u}}(\tau))^{2}\right\}}\right)
$$

which has a strong correlation with subjective perception.

Figs. 2 and 3 show the performance improvements by SDM and SBSD, respectively, in combination with no weighting (i.e., $w_{i}=1.0 \forall i$, dashed lines), and MUPA or the new MUPA-AK1 technique (solid lines). The curves for hard decision decoding (switch in position A in Fig. 1) are also depicted. As example, a maximum parameter SNR gain (vertical gain) of $2.61 \mathrm{~dB}$ at $E_{b} / N_{0}=2 \mathrm{~dB}$ is achieved by MUPA with hard decision decoding compared to the corresponding system with constant symbol energy. MUPA at the transmitter with hard decision decoding even outperforms systems using SDM or SBSD with AK0 at the receiver without MUPA (Fig. 2 for SDM and Fig. 3 for SBSD), although SDM and SBSD with AK0 need more computational effort for decoding the simple table lookup in the case of hard decision decoding.

With SDM and AK0, parameter SNR gains of 1.12 to $2.26 \mathrm{~dB}$ in the $E_{b} / N_{0}$ range of 1 to $6 \mathrm{~dB}$ are achieved

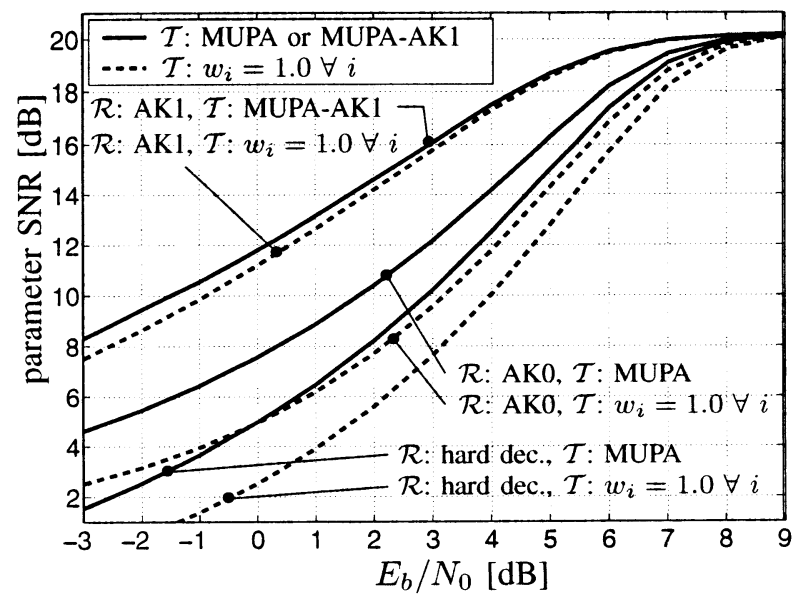

Fig. 2. Soft demodulation $(\mathrm{SDM}), \mathcal{R}$ : receiver site, $\mathcal{T}$ : transmitter site 


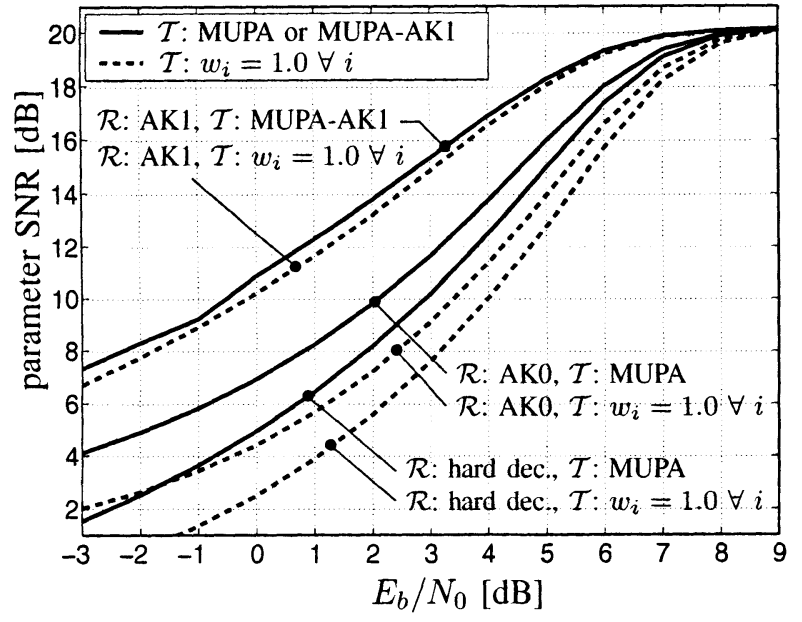

Fig. 3. Softbit source decoding (SBSD), $\mathcal{R}$ : receiver site, $\mathcal{T}$ : transmitter site

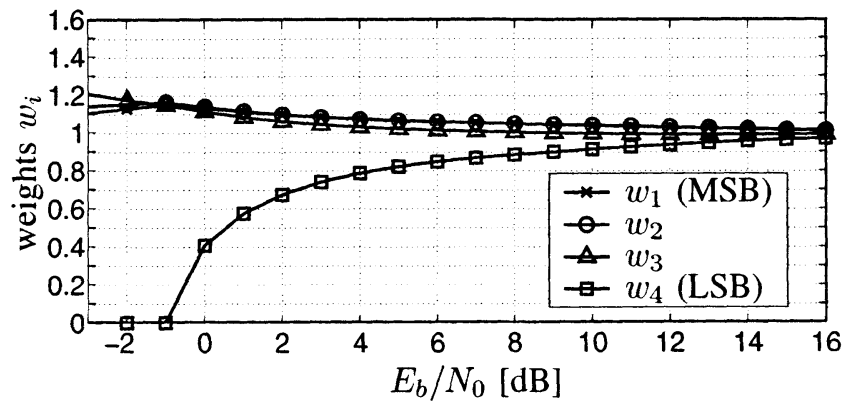

Fig. 4. MUPA-AK1 weight values $w_{i}$ (LMQ, $\Gamma$ : Gray)

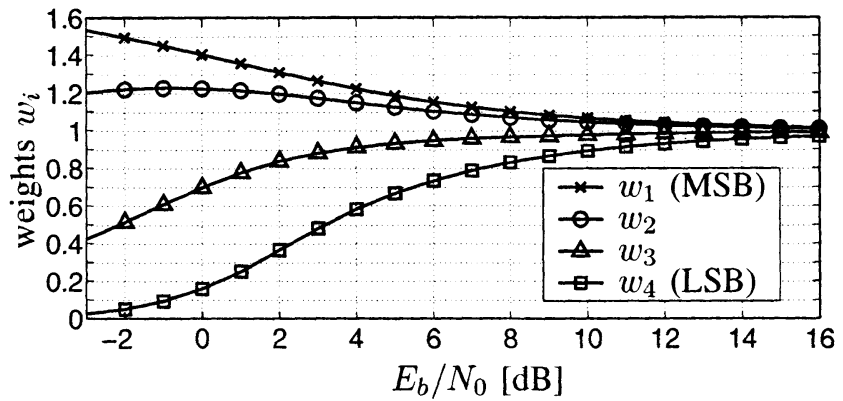

Fig. 5. MUPA weight values $w_{i}$ (LMQ, $\Gamma$ : Gray)

compared to systems with hard decision decoding (Fig. 2). MUPA combined with SDM and AK0 increases the parameter SNR by 1.35 to $2.7 \mathrm{~dB}$ in the $E_{b} / N_{0}$ range of 1 to $6 \mathrm{~dB}$ compared to the corresponding system without MUPA. Even if transmitter and receiver choose the wrong set of weights (within certain limits) due to imprecise estimation of the $E_{b} / N_{0}$, MUPA improves the parameter SNR, but with gains less than the maximum gains.

Fig. 2 shows a large performance improvement for systems using SDM with AK1 compared to a system with hard decision decoding: a maximum parameter SNR gain of $8.73 \mathrm{~dB}$ at $E_{b} / N_{0}=1 \mathrm{~dB}$. However, the novel MUPA-AK1 approach, which is developed in this paper and takes AK1 into account, even outperforms SDM with AK1. The gains in terms of parameter SNR are of 0.5 to $0.1 \mathrm{~dB}$ in the $E_{b} / N_{0}$ range of 1 to $5 \mathrm{~dB}$, while the maximum gain of $0.81 \mathrm{~dB}$ is reached at $E_{b} / N_{0}=-2 \mathrm{~dB}$. As the exploitation of AK1 at the receiver leads already to high parameter SNR values, the gains achieved by MUPA-AK1 are smaller than the ones for systems with AK0 or hard decision decoding as described above. With neg- ligible additional computational effort, MUPA-AK1 achieves a further improvement of systems with SDM and AK1. Note, that all weights $w_{i}$ can be calculated once in advance.

Analogously, Fig. 3 shows similar parameter SNR gains for systems using SBSD [5] instead of SDM at the receiver. A comparison of Figs. 2 and 3 shows that SDM outperforms SBSD. MUPA improves SBSD with AK0 by parameter SNR gains between 1.41 to $2.59 \mathrm{~dB}$ in the $E_{b} / N_{0}$ range of 1 to $6 \mathrm{~dB}$ compared to the corresponding system without MUPA. The parameter SNR gain of $7.75 \mathrm{~dB}$ achieved at $E_{b} / N_{0}=1 \mathrm{~dB}$ by SBSD with AK1 compared to systems with hard decision decoding is outperformed by the novel MUPA-AK1 by 0.65 to $0.24 \mathrm{~dB}$ in the $E_{b} / N_{0}$ range of 1 to $5 \mathrm{~dB}$.

Figs. 4 and 5 clarify the main difference between the weights $w_{i}$ of MUPA and MUPA-AK1. For $E_{b} / N_{0}>4 \mathrm{~dB}$ the MUPA-AK1 weights are closer together than the ones for MUPA. Consequently, MUPA causes a higher BER for the two LSBs than MUPA-AK1. For $E_{b} / N_{0}<4 \mathrm{~dB}$ the LSB weight $w_{4}$ decreases to 0 very fast in the case of MUPA-AK1.

\section{CONCLUSIONS}

The modulation with unequal power allocation (MUPA) varies the transmission power of each modulation symbol according to its significance and the channel quality by weights calculated once in advance. In this contribution, MUPA at the transmitter is applied for the first time to BPSK systems exploiting AK with SDM or SBSD at the receiver. In addition, the novel approach MUPA-AK1 is developed, which takes the autocorrelation of the source encoded parameters (i.e., AK1) into account. In the case of SDM and AK0, the parameter SNR gains by MUPA are between 1.35 and $2.7 \mathrm{~dB}$ in the $E_{b} / N_{0}$ range of 1 to $6 \mathrm{~dB}$ compared to systems with constant symbol energy. Even systems using SDM with AK1 are further improved by MUPA-AK1, but with smaller parameter SNR gains due to the already high parameter SNR values of SDM with AK1. MUPA at the transmitter has to be adapted to the specific receiver and the exploited a priori knowledge.

\section{REFERENCES}

[1] J. Hagenauer, "Rate-Compatible Punctured Convolutional Code (RCPC Codes) and their Applications," IEEE Transactions on Communications, pp. 389-400, Apr. 1988.

[2] T. Brüggen, C. Schulte-Hillen, and P. Vary, "Soft Demodulation and Unequal Error Protection for Digital Modulation Schemes," in Proc. of IEEE ICASSP, Philadelphia, PA, USA, Mar. 2005.

[3] T. Brüggen and P. Vary, "Analysis of Modulation with Unequal Power Allocation," in Proc. of IEEE VTC Spring, Stockholm, Sweden, May 2005.

[4] - "Unequal Error Protection by Modulation with Unequal Power Allocation," IEEE Commun. Lett., vol. 9, June 2005.

[5] T. Fingscheidt and P. Vary, "Softbit Speech Decoding: A New Approach to Error Concealment," IEEE Trans. Speech Audio Processing, pp. 240251, Mar. 2001.

[6] E. Bedrosian, "Weighted PCM," IRE Transactions on Information Theory, vol. IT-4, pp. 45-49, Mar. 1958.

[7] N. Görtz and E. Bresch, "Source-Adaptive Power Allocation for Digital Modulation," IEEE Commun. Lett., vol. 7, pp. 569-571, Dec. 2003.

[8] J. L. Melsa and D. L. Cohn, Decision and Estimation Theory. McGrawHill, 1978.

[9] J. G. Proakis, Digital Communications. McGraw-Hill Inc., 1995.

[10] A. Papoulis and S. U. Pillai, Probability, Random Variables, and Stochastic Processes. McGraw-Hill Inc., 2002. 